\title{
Modelado de las solicitaciones de los elementos estructurales de hormigón en edificios de gran altura en incendios reales
}

\author{
Modelling of the thermal solicitations of concrete structural \\ elements in tall buildings under natural fires
}

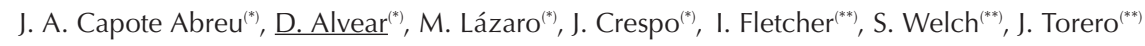

\section{RESUMEN}

El incendio de la Torre Windsor de Madrid constituye un suceso paradigmático de incendios en edificios de gran altura. En el presente Estudio se analizan las condiciones de origen, desarrollo y propagación de incendios reales en este tipo de edificaciones, así como la determinación de las condiciones de exposición (temperaturas $T$, flujos de calor $q^{\prime \prime}$, etc.) a las que se encuentran sometidos los elementos estructurales, en condiciones de uso final en este tipo de estructuras, mediante la utilización de técnicas de modelado y simulación computacional de incendios.

Los trabajos realizados, se centraron en aquellas actuaciones que: 1 ) permitieran validar la capacidad predictiva de los modelos de fluido-dinámica empleados, 2) la aplicación de los modelos ajustados y validados a un escenario de incendio en condiciones de uso final en un edificio de gran altura, para la predicción de la respuesta mecánica de la trama estructural.

\section{8-8}

Palabras Clave: modelado CFD, incendios en recintos cerrados, propagación interior, solicitaciones térmicas, respuesta estructural.

\section{SUMMARY}

The fire of the Windsor Building in Madrid represents a paradigm in High Rise Building Fires. The present Work analyzes the origin, growth and propagation conditions of natural fires in tall buildings. The Study has been focused on the determination of the thermal exposure conditions (temperatures $T$, heat fluxes $\dot{q}^{\prime \prime}$, etc.) on the structural members of high rise buildings, at end use conditions, under natural fires using fire computer modeling techniques.

Work allowed: 1) validate the predictive capacity of the fluid-dynamics computer models used, 2) apply these models to a specific fire scenario to assess the thermal and the mechanical response of the structural members of a high rise building.

Keywords: CFD modelling, room fires, fire spread, thermal loads, structural behaviour. 
1. Planta de Entrepiso del Edificio Windsor.

\section{INTRODUCCIÓN}

Cuando se examinan los efectos de incendios en un edificio utilizando herramientas de modelado y simulación computacional, se debe partir del hecho que, primeramente, el comportamiento de una estructura en caso de incendio está muy ligado al efecto que pueda tener el fuego sobre los elementos estructurales que la componen y segundo, que la redundancia dentro de la estructura puede permitir que las cargas se distribuyan aun cuando miembros estructurales aislados fallen.

En el presente trabajo, se tomó el incendio en el Edificio Windsor como base para realizar diferentes tipos de análisis: 1) determinación del inicio y desarrollo de las primeras etapas del incendio considerando como referencia el incendio en la oficina de origen (2109), y 2) la propagación interior en planta del incendio, seleccionando la planta 21. Esto permitió determinar la severidad alcanzada como consecuencia del incendio completamente desarrollado, debido a la combustión de los materiales presentes.

Posteriormente se realizaron cálculos mediante un modelo FEM (Método de los Elementos Finitos), usando las cargas térmicas calculadas para proveer de las condiciones de frontera de la fase-gas a los miembros estructurales. La utilización de este tipo de estudio permite calcular el impacto global de la temperatura dentro de la estructura, así como, mediante análisis más detallados, el comportamiento de los elementos dentro de la trama estructural.

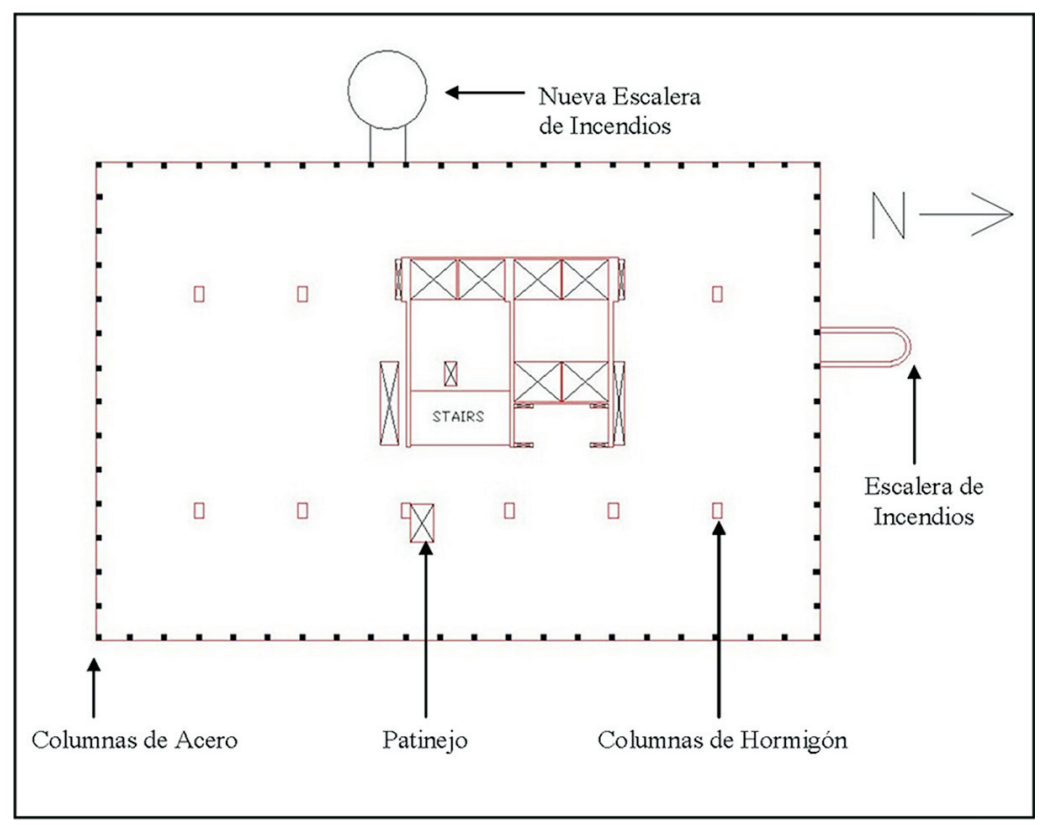

\section{DESCRIPCIÓN DEL EDIFICIO}

El 12 y 13 de febrero de 2005 el Windsor se vio envuelto en un incendio de 18 horas de duración, el cual comenzó en una oficina de la planta 21 causando un cuantioso daño estructural a las plantas superiores del edificio. Construido en el año 1978, a la fecha de su ejecución era uno de los edificios más altos de Madrid.

Estaba integrado en sus tres primeras plantas por un centro comercial y a partir de la tercera planta por una torre de planta rectangular compuesta por un núcleo y columnas portantes de hormigón, columnas perimetrales de acero y un forjado de entrepiso de losas alveolares con encofrado perdido de arcilla como muestra el esquema de la Figura 1.

En el momento del incendio, el edificio se encontraba en fase de ejecución de una mejora de la protección contra incendios ya que a la fecha de su construcción las columnas perimetrales de acero no contaban con protección ante incendios y además se había incluido una escalera adicional para la evacuación.

\section{MODELADO Y SIMULACIÓN COMPUTACIONAL DEL INCENDIO}

El modelado del incendio es una disciplina muy compleja debido al gran número de variables intervinientes. A la hora de aplicar una carga de fuego sobre la estructura, existen diferentes métodos (1), tal y como se describe a continuación.

La aproximación más sencilla al problema consiste en especificar una temperatura uniforme en la superficie de los elementos estructurales. Esta temperatura puede ser estimada de datos experimentales o de observaciones, teniendo en cuenta por ejemplo el color de las Ilamas o las condiciones post-incendio del material expuesto. En el caso de la Torre Windsor, hay evidencias consistentes de que las temperaturas de la fase-gas alcanzaron entre los $800{ }^{\circ} \mathrm{C}$ y $1.000^{\circ} \mathrm{C}$ después del flashover.

A falta de mediciones y observaciones, es posible realizar aproximaciones de las condiciones del desarrollo del incendio mediante la utilización de herramientas de modelado y simulación computacional de incendios. Dentro de estos métodos, existen diversos modelos los cuales se basan en simulaciones "de estado de equilibrio" y simulaciones transitorias.

En el presente estudio, el Modelo de Simulación Computacional de Incendios empleado para la realización de los trabajos fue el 'Fire Dynamics Simulator (FDS)', versión 4 (2), desarrollado por el Building and Fire Research Laboratory del National Institute of Standards and Technology - NIST (USA) con 
la colaboración del VTT Building and Transport in Finland.

FDS es un modelo de dinámica de fluidos computacional (CFD) concebido de manera específica para reproducir el fenómeno del incendio, el cual se encuentra ampliamente difundido y validado $(3,4)$ dentro de la comunidad científica internacional como herramienta análisis y predicción de la evolución de incendios en recintos cerrados. FDS resuelve numéricamente un tipo de ecuaciones de Navier-Stokes apropiadas para baja velocidad y flujo, considerado desde un punto de vista térmico con énfasis en el transporte de humo y calor derivados de incendios, [1], [2], [3], [4], [5].

$$
\frac{\partial \rho}{\partial t}+\nabla \cdot \rho \vec{u}=0
$$

$\frac{\partial \rho Y_{l}}{d t}+\vec{u} \cdot \nabla \rho Y_{l}=-\rho Y_{l} \nabla \cdot \vec{u}+\nabla \cdot \rho D_{l} \nabla Y_{l}+\dot{m}_{l}^{\prime \prime}$

$\frac{\partial}{\partial t}(\rho \bar{u})+\nabla \cdot \rho \bar{u} \bar{u}=\nabla p+\rho \bar{f}_{T}+\nabla \cdot \tau_{j}$

$\frac{\partial}{\partial t}(\rho h)+\nabla \cdot \rho h \bar{u}=\stackrel{P}{D}+\dot{q}^{\prime \prime \prime}-\nabla \cdot \bar{q}_{r}+\nabla \cdot k \nabla T+$

$$
\begin{gathered}
+\nabla \cdot \sum_{l} h_{l}(\rho D)_{l} \nabla Y_{l} \\
p_{0}=R \rho T \sum_{l} \frac{Y_{l}}{M_{l}}
\end{gathered}
$$

Donde: [1] Ec. Conservación de la Masa, [2] Ec. Conservación de las Especies, [3] Ec. Conservación de Momentum (Navier-Stokes), [4] Ec. Conservación Energía, [5] Ec. de Estado.

El algoritmo principal tiene un esquema predictivo-corrector explícito de segundo orden de exactitud en el espacio y en el tiempo. La turbulencia es tratada por medio de la forma Smagorinsky de Simulación de Grandes Remolinos (Large Eddy Simulation, LES).

\subsection{Análisis de validación de las capacidades predictivas del modelo}

Previo a la realización del análisis del escenario de incendio planteado, se desarrolló un proceso de validación de las capacidades predictivas del Modelo de Simulación Computacional escogido, para lo cual se tomó como referencia los Ensayos de Fuego a escala real realizados en un edificio de gran altura en Dalmarnock (Glasgow, UK), estudio liderado por la Universidad de Edimburgo (UK) (4).

Los resultados arrojados por las simulaciones mostraron una gran disparidad entre la predicción de los modelos de simulación y las mediciones experimentales, no obstante, los resultados obtenidos en las simulaciones del comportamiento general del incendio son lo suficientemente fiables para ser utilizados en análisis simplificados de ingeniería.

A fin de mejorar las capacidades predictivas del modelo, han sido analizadas previamente las variabilidades de las salidas obtenidas con FDS y se han mejorado los parámetros introducidos al modelo para pulir al máximo los cálculos de la dinámica del incendio (5). Así mismo, se estudió la influencia de la turbulencia y el refinamiento espacial en la exactitud de los resultados $(6,7)$.

Dichos análisis han permitido llegar a consensos sobre la importancia de la correcta selección de los parámetros de entrada en el modelo y el refinamiento espacial de la malla en la exactitud de los resultados de los cálculos de la dinámica del incendio.

\subsection{Desarrollo del incendio en la oficina de origen}

Antes de tratar de establecer un estudio que permitiera comprender el desarrollo del incendio a través del interior del edificio, se decidió focalizar la atención en cómo pudo ser el desarrollo del mismo en la habitación en la que éste se inició y en el cálculo de la velocidad de cesión de calor total que se produjo en la misma.

Los elementos técnicos resultantes de este análisis, además de permitir continuar con el estudio en toda la planta y entre plantas, facilitaron poder avalar la hipótesis de origen del incendio, y determinar la importancia de los diferentes factores que influyen en su crecimiento en un recinto cerrado: dimensiones del recinto, potencia de la fuente de ignición, características, distribución y tipos de elementos combustibles, condiciones de ventilación, etc.

El modelo computacional de la oficina de origen se construyó de una manera versátil, de forma que se pudiesen variar fácilmente las características de los materiales y otras condicionantes (ej. tiempo de rotura de las ventanas) para estudiar la influencia de las mismas en el progreso del incendio (Figura 2).
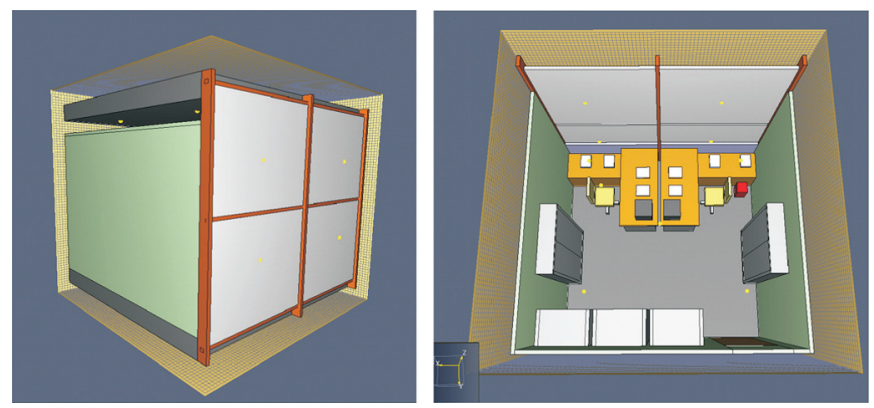

2. Modelo computacional del recinto de origen del incendio. 
La focalización del Estudio en esta primera fase únicamente en la habitación 2109 hizo posible la utilización de un mallado con un importante grado de refinamiento, permitiendo resolver con elevada precisión las ecuaciones de Navier-Stokes. De este modo, el dominio computacional del despacho resultó dividido en un total de 512.000 celdas.

Una vez creado el dominio computacional, se definieron las características de los

Tabla 1. Características de los materiales de revestimiento

\begin{tabular}{|c|c|c|c|c|c|}
\hline Ref. & $\begin{array}{c}\text { Espesor } \\
(\mathrm{mm})\end{array}$ & $\begin{array}{c}\text { Densidad } \\
\left(\mathrm{Kg} / \mathrm{m}^{2}\right)\end{array}$ & $\begin{array}{c}\text { Calor Esp. } \\
\left(\mathrm{KJ} / \mathrm{Kg}^{\circ} \mathrm{K}\right)\end{array}$ & $\begin{array}{c}\text { Tig } \\
\left({ }^{\circ} \mathrm{C}\right)\end{array}$ & $\begin{array}{c}\text { HRR pico } \\
\left(\mathrm{kW} / \mathrm{m}^{2}\right)\end{array}$ \\
\hline Paredes $(5)$ & 9,5 & 440 & 1,47 & 326 & 243,36 \\
\hline Suelo & 6 & 750 & --- & 290 & 374 \\
\hline Falsos techos & 13 & 1.440 & --- & 325 & 38,92 \\
\hline
\end{tabular}

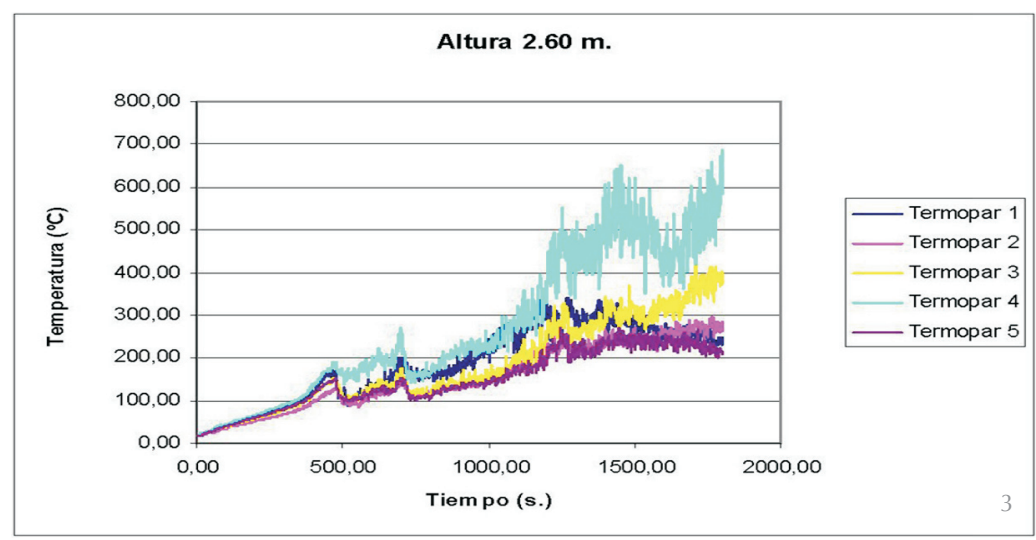

3. Temperaturas registradas por os termopares dentro de la oficina 2109 .

4. Propagación del incendio en la planta de origen
Una vez introducidas las propiedades de los materiales de recubrimiento al modelo, se procedió a implementar las características del mobiliario presente en la oficina, para los cual se utilizaron los resultados de ensayos del NIST en el estudio "Two Panel Workstation Fire Test" (9) y posteriormente fueron definidas las características de la fuente de ignición, utilizándose para el estudio una papelera.

Las condiciones de ventilación implementadas en el modelo están determinadas por el momento de la rotura de los cristales, considerándose dos condiciones de ventilación: una primera donde los cristales se rompieron tras alcanzarse una temperatura de $150^{\circ} \mathrm{C}$, y una segunda donde se eliminaron los cristales desde un primer momento.

Una vez implementados todos los parámetros en el modelo, se procedió al cálculo del desarrollo de la dinámica del incendio en el recinto. La Figura 3 muestra los valores de temperatura registrados por los termopares bajo cubierta para la condición más desfavorable durante los primeros 1.800 segundos del desarrollo de la simulación del incendio.

Los resultados de este primer análisis demostraron que era posible alcanzar estadios de incendio completamente desarrollado en el despacho a partir de pequeñas fuentes de ignición, tales como la papelera.

Durante el desarrollo del incendio se comprobó la ignición de forma generalizada en el ala de la mesa que se encuentra ubicada al lado de la papelera antes de los $30 \mathrm{~min}$, la rotura de los cristales superiores en este intervalo de tiempo, así como la propagación del incendio a los recintos adyacentes al despacho 2109 por medio de la pared más cercana a la papelera.

\subsection{Desarrollo del incendio en la planta 21}

La planta típica de la Torre Windsor estaba estructurada en una serie de oficinas perimetrales divididas a través de paneles de aglomerado de madera, una serie de puestos de trabajo en la parte central izquierda y de un núcleo central de hormigón en el que se localizan los ascensores, las escaleras, los baños y una serie de salas de reuniones.

Los estudios realizados en la planta se focalizaron en dos objetivos específicos, por un lado 1) estudiar y analizar el desarrollo del incendio en esa planta y por otro 2) permitir el cálculo de curvas paramétricas de velocidad de cesión de calor total representativas de incendios reales completamente desarrollados en planta.

Como se puede apreciar en la Figura 4, el desarrollo del incendio se produjo tanto a 
través de las paredes como por el pasillo. Se desarrollaron diferentes simulaciones del desarrollo del incendio bajo diferentes condiciones de ventilación como se realizó para la oficina donde se inició el incendio, lo cual demostró ser un factor crítico en la evolución del mismo.

En este sentido, se constató la necesidad de tener especial atención con las condiciones de agotamiento del oxígeno debido al rápido crecimiento del incendio, primero en la habitación y posteriormente en el resto de la planta. Esta falta de oxigeno no se produjo en los laterales donde se rompieron las cristaleras.

A partir de los resultados obtenidos en las simulaciones, se calcularon las curvas de velocidad de cesión de calor total que se produjeron en la planta para las condiciones analizadas. Este dato proporcionó un indicador de la magnitud y severidad del incendio y resultó de gran interés en el análisis de las solicitaciones térmicas en los elementos estructurales.

Se realizó un análisis para comprobar los resultados proporcionados por curvas paramétricas de velocidad de cesión de calor, en relación con las condiciones de ataque térmico (temperatura, flujos de calor, etc.) suficientes para hacer que la estructura colapsase.

A efectos de simplificar el proceso de cálculo, se simplificaron las condiciones de uso final de la planta 21, considerando únicamente los elementos estructurales junto con unos incendios de diseño representativos de la velocidad de cesión de calor anteriormente calculadas. Las curvas paramétricas de velocidad de cesión de calor se introdujeron en forma de incendio de diseño, situado en toda la superficie del suelo de la planta.

Se estudiaron diferentes curvas paramétricas mostrándose las que condujeron condiciones extremas como se muestra en la Figura 5. La primera curva cuenta con un crecimiento cuadrático hasta aproximadamente $225 \mathrm{MW}$.

La segunda curva paramétrica seleccionada fue de aproximadamente el doble de la anterior en el valor de pico (500 MW), con el propósito de considerar una situación extremadamente severa (debido, por ejemplo, a las incertidumbres del proceso de modelado). Debe precisarse que el tamaño de esta última curva excede deliberadamente los límites superiores aproximados de un incendio controlado por ventilación en toda la planta, obtenidos con la expresión $\left[\dot{m}=5.5 A_{w} \sqrt{h}\right](\mathrm{kg} / \mathrm{min})$, los cuales son del orden de $350 \mathrm{MW}$. En cualquier caso, cuando la altura de la apertura de ventilación, h, es igual a dos metros, este valor se torna del orden de $493 \mathrm{MW}$, aproximadamente los 500 MW considerados.
Posteriormente se aplicaron las curvas paramétricas obtenidas al modelo de FDS y tal como cabía esperar debido a las magnitudes de incendios completamente desarrollados como los seleccionados en las curvas paramétricas, las temperaturas ambientales que produjeron
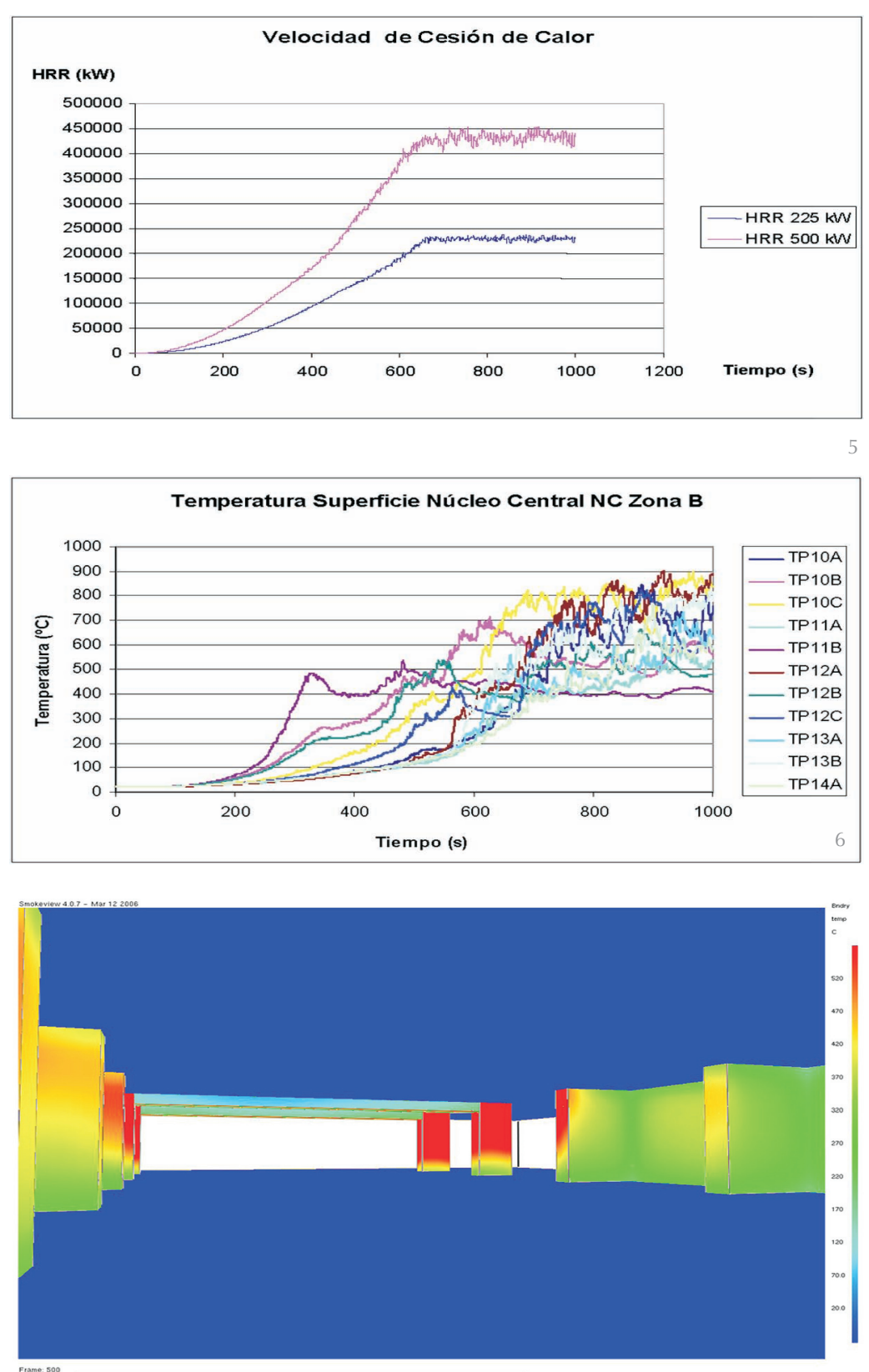

Fiane soo

estas curvas de diseño fueron de un valor considerable, alcanzándose valores de hasta 1.160 ${ }^{\circ} \mathrm{C}$ cuando la curva de velocidad de cesión de calor es de $500 \mathrm{MW}$.

La Figura 6 muestra los resultados de las temperaturas registradas por los termopares durante las simulaciones en la zona del núcleo central del edificio. Los valores máximos alcanzados por la temperatura dentro de la planta se encuentran en concordancia con los valores estimados tras la evaluación de la estructura. La Figura 7 mues- 
tra la visualización del ataque térmico realizado a los elementos estructurales tras aplicarle al modelo la curva paramétrica de velocidad de cesión de calor de 500 MW.

A partir de estos resultados, es posible su consideración como valores de entrada en un programa de elementos finitos para determinar la respuesta de la estructura, proceso que suele ser complejo y que se explica más al detalle a continuación.

\section{MODELADO Y SIMULACIÓN DEL COMPORTAMIENTO ESTRUCTURAL}

En muchos casos, no es necesario ni deseable basar la geometría del modelo en la totalidad de la estructura. Un dominio más simplificado, consistente en una o varias plantas, proporciona una gran cantidad de información que puede ser extrapolada al resto de la edificación (10).

Cuando examinamos modelos de propagación de incendios, incluyendo aquellos de propagación vertical de las llamas, el principal foco de interés recae en la magnitud y el tiempo en que los elementos individuales están sujetos a la exposición térmica. Aunque un modelo de una planta completa puede proporcionar esta información, si se conoce que los efectos direccionales del viento no fueron relevantes, como en el presente caso de estudio, es posible emplear planos simétricos para reducir el tamaño del dominio. Es razonable asumir que si la planta se divide en cuartos a través de ejes de simetría entonces cada cuarto de la planta va a estar afectado de manera similar (tiempos de flashover y extinción, por ejemplo), por lo que estas técnicas serán empleadas en análisis posteriores de propagación vertical en siguientes estudios.

De la misma forma, al crear un modelo de elementos finitos de la estructura, de nuevo puede ser poco ventajoso crear un modelo tridimensional de la planta completa. Igualmente para reducir el número de elementos necesario, donde sea posible, en vez de utilizar elementos tipo sólido, se pueden utilizar elementos tipo "beam", "shell" o "membrane". Además, el modelo puede ser simplificado tomando un plano de corte de la sección de la planta y analizándola como una viga, como es práctica común en ingeniería estructural.

Mientras esta aproximación puede excluir contabilizar muchas de las redistribuciones de carga que comúnmente tienen lugar en una estructura tridimensional, puede ser utilizada para, en modelos simplificados, examinar factores como el fallo de un elemento individual dentro de la estructura. Por ejemplo, el efecto del fallo de los pilares de acero sin protección contra el fuego sobre el forjado de entrepiso puede ser examinado simplificando el forjado como una viga extendida entre el núcleo de hormigón y la columna de acero.

\subsection{Modelado del hormigón durante el incendio}

En el modelado por elementos finitos del hormigón armado como miembro estructural existen ciertas propiedades inherentes al material que requieren una cuidadosa atención. En primer lugar, el hormigón es un buen aislante, por tanto la penetración térmica dentro del miembro será baja. Así mismo, el hormigón armado es un material compuesto consistente tanto en hormigón como en el acero de refuerzo. Las propiedades tanto del hormigón como del acero de refuerzo son dependientes de la temperatura, y cuando cargamos una viga de hormigón armado se comportará primero elásticamente y luego plásticamente después de ceder.

\subsection{Modos de fallo de la Torre Windsor}

Existen un gran número posible de modos de fallo para la Torre Windsor, y es probable que una variedad de estos ocurrieran en la práctica. Algunos de estos son:

1. Colapso del entrepiso a causa del fallo previo de las columnas de acero del perímetro. Esta hipótesis supone que el entrepiso se vio forzado a trabajar como un voladizo partiendo del núcleo.

2. Colapso del forjado entre el núcleo y los pilares exteriores al núcleo. Esta hipótesis supone que producto de los grandes espacios vacíos existentes en esta zona por encontrarse los patinillos, el área del entrepiso capaz de soportar las grandes fuerzas de tracción que se originaron por el fallo del entrepiso exterior a los pilares no era suficiente.

La naturaleza multi-pisos del incendio es indudablemente importante, y es probable que si el incendio sólo se hubiese concentrado en una planta, la mayoría del edificio hubiese sobrevivido intacto. Una evidencia a este planteamiento se encuentra en el robusto comportamiento sobre la novena planta, donde el fallo local de los pilares perimetrales de acero fue reacomodado mediante redistribución local.

\subsection{Modelado estructural de la Torre Windsor}

El modelado del comportamiento de una estructura completa es una tarea muy compleja, y es a menudo deseable empezar con elementos individuales de la estructura y después incrementar el nivel de complejidad 
del modelo. En el caso de la Torre Windsor, el primer elemento estructural a modelar fue el forjado dado que la naturaleza de la construcción del entrepiso, hace que este no puede ser simplemente modelado como una membrana soportada por vigas.

Examinando los planos, resulta evidente que el forjado actúa como una red de vigas. Esto puede ser modelado como vigas primarias que soportan vigas secundarias. Sin embargo, las vigas primarias y secundarias no necesariamente se extienden en la misma dirección, esto quiere decir que, mientras en una sección del edificio las vigas principales corren de norte a sur, en otra sección corren de este a oeste.

Es también necesario hacer suposiciones acerca de las condiciones de frontera en el modelo de la viga. Donde la viga se une con el núcleo de hormigón del edificio se puede asumir que existe empotramiento de la viga mientras que en la unión con el pilar podemos asumir que la conexión es una articulación. A pesar de que la columna está embebida en el forjado, el tamaño relativamente pequeño de ésta $(140 \times 120 \mathrm{~mm})$ da a entender que es improbable que resista cualquier momento trasmitido del forjado (Figura 8).

Una vez que ha sido definida la estructura, es necesario examinar el efecto del calentamiento sobre ella, específicamente la evolución del calentamiento dentro del material, es decir, el grado de penetración del flujo térmico en el elemento de hormigón estructural y las temperaturas a las cuales ha sido expuesto el acero de refuerzo. Esto hace necesario examinar el perfil de temperatura dentro de la sección de la viga.

La herramientas utilizadas para el cálculo estructural mediante FEM fueron los modelos ABAQUS, SAP2000 y SAFE. Aunque pudiese pensarse que un elemento tipo beam se podría utilizar para modelar las vigas primarias, las limitaciones para representar los efectos térmicos dan a entender que es más práctico utilizar elementos tipo shell. Con esto, la temperatura puede definirse a un número mayor de profundidades dentro del elemento. La viga de hormigón ha sido por tanto modelada como un elemento shell estrecho y profundo.

\subsection{Análisis}

Los modelos de desarrollo del incendio en la planta donde se originó el mismo han sido descritos anteriormente por (11), focalizándose estas simulaciones en la atención al aspecto del fallo de ventilación con los dos casos expresados.

También se han considerado las incertidumbres en las características y la distribución de los materiales combustibles, por lo que los cálculos fueron procesados con velocidades de suministro de combustible que se corresponden aproximadamente a dos veces lo que se podría esperar para tener una ven-

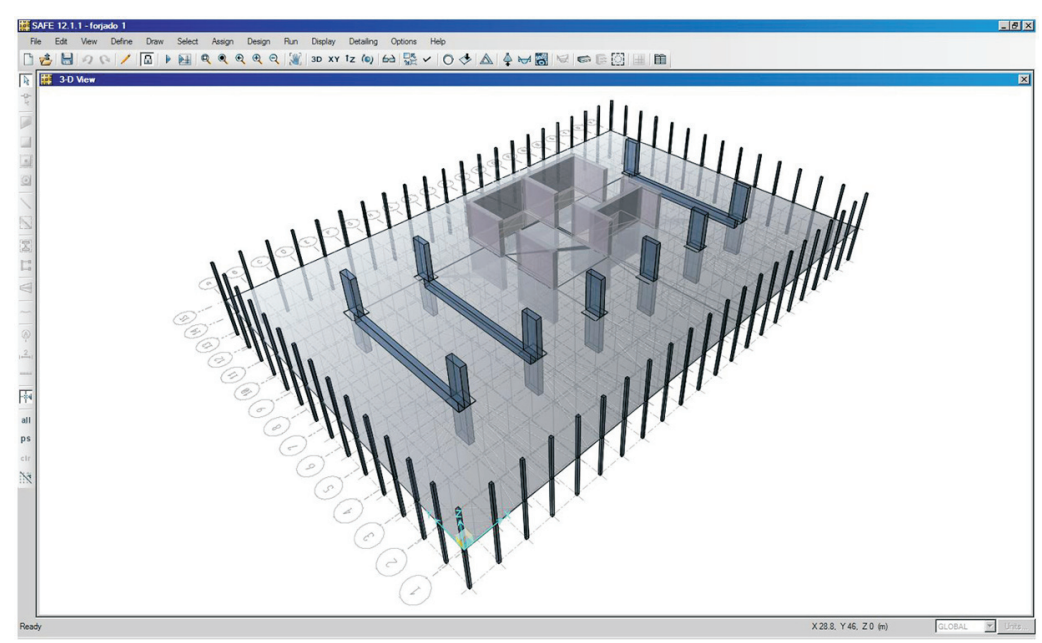

tilación controlada [ $\dot{m}=5.5 A_{w} \sqrt{h}$ ], con una velocidad de cesión de calor por piso de 350 $\mathrm{MW}$, basado en la suposición de una altura de ventana de la mitad de la distancia del suelo al techo de $3 \mathrm{~m}$.

Mientras transcurría el incendio, pasado algún tiempo, algunos de los paneles de aluminio localizados en la base de las aperturas se perdieron, dando acceso a aun más ventilación, pero en esta etapa la carga de combustible del incendio puede haber sido sustancialmente disminuida debido a su consumo.

Basados en estas suposiciones de ventilación, las temperaturas máximas calculadas de la fase-gas exceden los $1.100{ }^{\circ} \mathrm{C}$ y las temperaturas correspondientes en la superficie de los pilares de hormigón fueron aproximadamente $200{ }^{\circ} \mathrm{C}$ menores.

Aquí existen pequeñas variaciones de esta predicción respecto al tamaño del incendio previsible, lo cual estuvo condicionado a partir del momento en que el límite de ventilación controlada fue excedido y el combustible extra ardió principalmente fuera del compartimiento.

Estas estimaciones están además soportadas por el análisis post-incendio de las resistencias del hormigón (12), que confirmaron que la temperatura en la superficie de las columnas y forjados había excedido los 800 ${ }^{\circ} \mathrm{C}$. Es también posible hacer un estimado de las temperaturas en la superficie del hormigón basado en la metodología de examinar los cambios de coloración en el hormigón y los cambios en la microestructura interna. 
9. Deflexiones en la Planta 21.

10. Profundidad de penetración térmica contra el tiempo.
Examinando el comportamiento de la estructura usando los códigos del FEM, un dato muy importante es la entrada de las condiciones de temperatura en la estructura. Los programas de elementos finitos como ABAQUS pueden por supuesto calcular la respuesta térmica basada en la exposición para las condiciones de borde conocidas, tanto temperaturas como flujos, pero normalmente se necesita el uso de elementos tipo bloque, los cuales son ineficientes en la modelación estructural. Por tanto, la respuesta térmica ha sido examinada usando modelos térmicos independientes anteriormente descritos. Usando elementos tipo shell, se especifican distintas temperaturas a cada capa, y por lo tanto las barras de refuerzo. Una consideración importante aquí es la profundidad del elemento en relación a la profundidad del flujo térmico que se propaga dentro del material.

Si este último es muy superficial, entonces la distribución de la temperatura dentro de la estructura no podrá ser adecuadamente representada a menos de que existan suficientes nodos para resolverlo. Si la cantidad

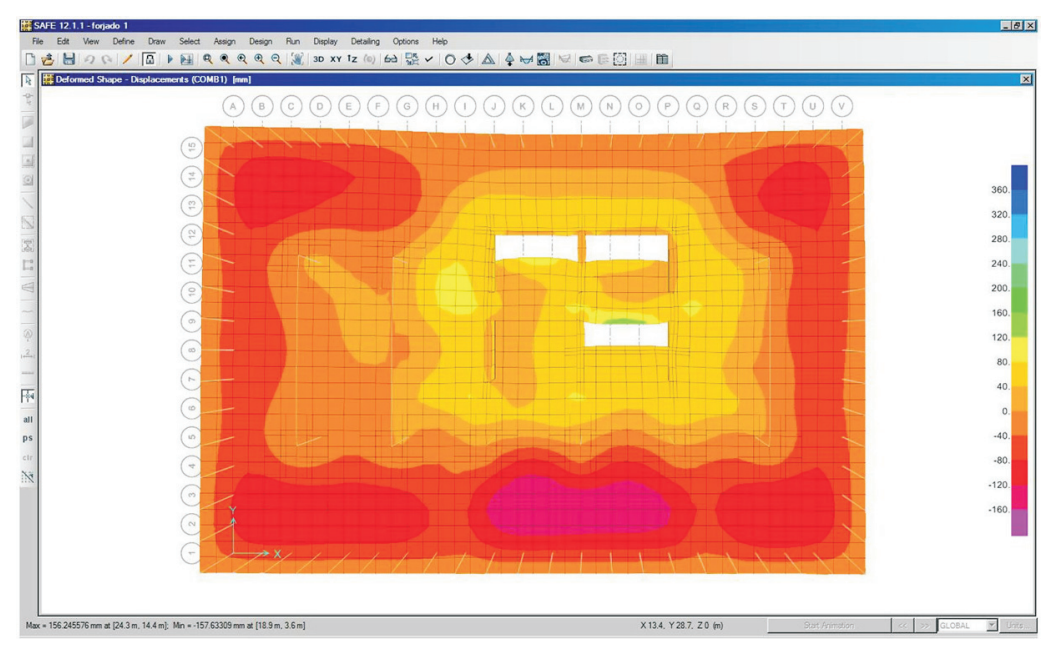

PROFUNDIDAD DE PENETRACIÓN TÉRMICA EN EL HORMIGON

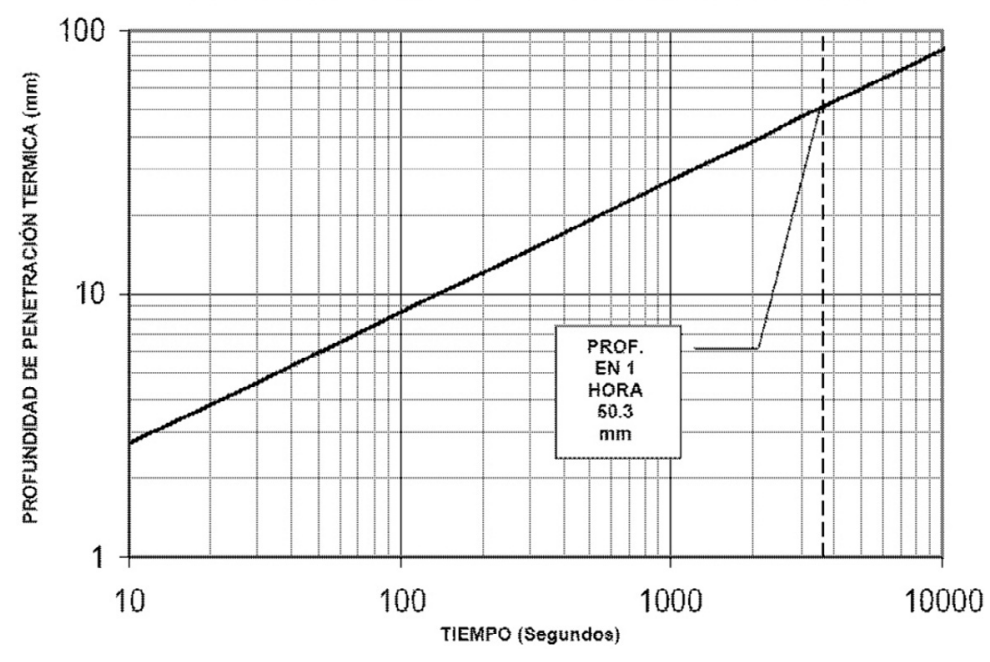

de nodos son insuficientes, el efecto del calentamiento tenderá a ser exagerado y esto podría tener un efecto que repercutirá en el modelo mecánico.

Por tanto, con el fin de mostrar esta modelación se ha realizado un cálculo sencillo de la evolución de la penetración térmica frente al tiempo. La resistencia especificada para la estructura fue 24,5 MPa en los pilares y paredes, 29,4 MPa en las vigas y 17,2 MPa en el forjado de entrepiso, aunque hubo algunas variantes al respecto en la práctica (13). Considerando un hormigón normal de agregados silicios, la densidad fue tomada como $2.400 \mathrm{~kg} / \mathrm{m}^{3}$, la conductividad térmica como $1,2 \mathrm{~W} / \mathrm{m} / \mathrm{K}$ y el calor específico como $880 \mathrm{~J} / \mathrm{kg} / \mathrm{K}$, dando una difusión térmica de $0,57 \times 10^{-6} \mathrm{~m}^{2} / \mathrm{s}$.

La Figura 9 muestra los resultados del patrón de deflexiones del modelo para una de las condiciones de ataque térmico estudiadas. Los resultados obtenidos concuerdan con el modo de fallo 1 comentado anteriormente. La Figura 10 muestra la evolución de la penetración térmica en la profundidad a lo largo del tiempo. Los datos de la duración del incendio no fueron completamente precisados, pero existen evidencias que las fases principales de éste duraron alrededor de una hora (14); arrojando unos resultados de penetración de alrededor de $50 \mathrm{~mm}$.

Comparando con estudios realizados a la resistencia del hormigón después del fuego sugieren que en pequeñas regiones del techo, basado en el criterio de la isoterma $500{ }^{\circ} \mathrm{C}$, había excedido una profundidad de 200 mm (15). En este caso puede haber por supuesto efectos transversales incluyendo calentamiento proveniente de las caras de las vigas que conforman el forjado reticular (dimensiones de 100x200 mm, excluyendo la losa superior).

Usando por defecto un elemento tipo shell de 5 nodos, el espaciamiento en el modelo de la viga de $230 \mathrm{~mm}$ de altura y el acoplamiento con la losa de $58 \mathrm{~mm}$, la onda térmica recorre el forjado en aproximadamente $1.400 \mathrm{~s}$. La Figura 10 sugiere que al menos en los 100 primeros segundos sea usada una resolución de $10 \mathrm{~mm}$ para poder describir adecuadamente la respuesta térmica del forjado en la simulación.

\section{CONCLUSIONES}

La fase inicial de los trabajos de investigación ha mostrado la capacidad de los Modelos de Simulación Computacional de Incendios para realizar el análisis del desarrollo de un incendio dentro de tanto un recinto pequeño, como una habitación, como de un recinto más grande como puede ser la planta completa. 
Se ha mostrado, en términos cualitativos, como el incendio puede crecer y desarrollarse desde la habitación origen del incendio, lo que proporcionó una base para establecer una representación del posible desarrollo del incendio en el piso completo. Estos modelos permitieron además de analizar el desarrollo del incendio, y calcular las cargas térmicas a las que se vio sometida la estructura. Estas cargas térmicas podrán emplearse en fases posteriores para analizar la respuesta mecánica (tensiones y deformaciones) de la estructura en interacción con modelos de elementos finitos (MEF).

Se ha realizado un enfoque global para valorar el comportamiento de la estructura principal de hormigón de la Torre Windsor. Esto ha permitido realizar una valoración de los registros de exposición térmica más probables, consultando datos obtenidos de la valoración de la estructura posterior al incendio y varios métodos basados en los modelos de simulación computacional del incendio. La respuesta térmica de la estructura fue posteriormente evaluada, con el fin de definir los datos de partida y las condiciones de frontera para los modelos estructurales incluyendo FEM. Basado en esto, se ha desarrollado una estrategia para modelar la respuesta mecánica de la estructura, con vista de analizar los posibles mecanismos de fallo en relación con los efectos observados del incendio.

\section{AGRADECIMIENTOS}

Los autores desean agradecer el apoyo del Ministerio de Educación y Ciencia (ref. BIA 2006-00388). Así mismo, se expresa el agradecimiento al Dr. Guillermo Rein por su colaboración y apoyo.

\section{BIBLIOGRAFÍA}

(1) Jowsey, A.: "Fire Imposed Heat Fluxes for Structural Analysis", PhD thesis, University of Edinburgh, UK. 2006.

(2) McGrattan, K.: "Fire Dynamics Simulator (Version 4) - Technical Reference Guide Volume 1: Mathematical Model", National Institute of Standards and Technology (NIST). 2005.

(3) McGrattan, K.: "Fire Dynamics Simulator (Version 4) - Technical Reference Guide Volume 3: Validation", National Institute of Standards and Technology (NIST). 2005.

(4) Rein, G.; Torero, J.L.; Jahn, W.; Stern-Gottfried, J.; Ryder, N.L.; Desanghere, S.; Lázaro, M.; Mowrer, F.; Coles, A.; Joyeux, D.; Alvear, D.; Capote, J.A.; Jowsey, A.; Abecassis-Empis, C.; Reszka, P.: "Round-Robin Study of a priori Modelling Predictions of The Dalmarnock Fire Test One" Fire Safety Journal, Vol. 44 Issue 4, 590-602. 2009. doi: 10.1016/j.firesaf.2008.12.008.

(5) Lazaro, M.; Bohmer, H.; Alvear, D.; Capote, J.A.; Trouve, A.: "Numerical Simulation of Fire Growth, Transition to Flashover, and Post-Flashover Dynamics in the Dalmarnock Fire Test". Fire Safety Science 9: 1377-1388. 2008

(6) Lazaro, M.: "Influencia de la Discretización Espacial en la Exactitud del Modelado de Fluidodinámica Computacional de Incendios", Tesis Doctoral, Universidad de Cantabria, 2008.

(7) Capote, J.A.; Abreu, O.V.; Alvear, D.; Lázaro, M.; Espina, P.: "Influencia del Modelo de Turbulencia y del Refinamiento de la Discretización Espacial en la Exactitud de las Simulaciones Computacionales de Incendios" Revista Internacional de Métodos Numéricos para el Cálculo y Diseño en Ingeniería, Vol. 24, No 3, 227-245. 2008

(8) Madrzykowski, D.; Walton, W.D.: "Cook County Administration Building Fire, 69 West Washington, Chicago, Illinois, October 17, 2003: Heat Release Rate Experiments and FDS Simulations", Building and Fire Research Laboratory, National Institute of Standards and Technology, NIST Special Publication SP-1021, July 2004.

(9) NIST Standard Reference Database Number 75, January 1999 Release

(10) FIRESTARR Final Report. CEN TC256 / CENELEC TC9x JWG Fire protection. 2001.

(11) Flint, G.: "Fire induced collapse of tall buildings.", PhD thesis, University of Edinburgh, UK. 2005.

(12) Kono, M.; et. ál.: "The Madrid city Windsor building fire inspection report.", p. 133, The Madrid city Windsor building fire investigation committee (BCS, JSCA et ál) (In Japanese), 2005.

(13) Capote, J.A.; Alvear, D.; Lázaro, M.; Espina, P.; Fletcher, I.A.; Welch, S.; Torero, J.L.: "Analysis of thermal fields generated by natural fires on the structural elements of tall buildings", Proc. Int. Cong. Fire Safety in Tall Buildings. Santander, Spain. 2006.

(14) Fletcher, I.A.; Welch, S.; Capote, J.A.; Alvear, D.; Lázaro, M.; Espina, P.: "Model-based analysis of a concrete building subjected to fire", Proc. Advanced Research Workshop Fire Computer Modeling. Santander, Spain. 2007.

(15) Calavera, J.; González-Valle, E.; Díaz-Lozano, J.; Fernández-Gomez, J.; Ley, J.; Izquierdo, J.M.: "Fire in the Windsor building, Madrid. Survey of the fire resistance and residual bearing capacity of the structure after the fire". Instituto Técnico de Materiales y Construcciones (INTEMAC). 2005. 